\title{
The Nutritional Requirements of some Pasteurella Species
}

\author{
By T. W. BURROWS AND W. A. GILLETT \\ Microbiological Research Establishment, Porton Down, \\ Salisbury, Wiltshire
}

(Received 11 March 1966)

\begin{abstract}
SUMMARY
The nutritional requirements for growth on agar media at $28^{\circ}$ and at $37^{\circ}$ were investigated for strains representative of Pasteurella pseudotuberculosis, $\boldsymbol{P}$. pestis and Pasteurella strain ' $\mathrm{X}$ ' (Yersinia enterocolitica). At 28', strains of $\boldsymbol{P}$. pseudotuberculosis either required no growth factors or were dependent on thiamine or pantothenate. Most strains of $\boldsymbol{P}$. pestis required cystine, methionine and phenylalanine but some strains could dispense with methionine or phenylalanine while others required additional factors. Strains of Pasteurella ' $\mathbf{X}$ ' either were thiamine-dependent or required no factors. At $37^{\circ}$ all strains showed additional requirements. Most strains of $\boldsymbol{P}$. pseudotuberculosis could then grow with any 3 of the 4 factors glutamic acid, thiamine, cystine and pantothenate; other strains specifically required all 4 factors and nicotinamide. Strains of $\boldsymbol{P}$. pestis grew reliably on media supplemented with cystine, methionine, phenylalanine, glycine, valine, isoleucine, glutamic acid and thiamine when incubated in $\mathrm{CO}_{2}$ enriched air. All strains of Pasteurella ' $X$ ' required thiamine and either cystine or methionine; some substrains showed additional requirements. Virulent and avirulent representative strains of $P$. pestis had similar calcium requirements for growth on defined media.
\end{abstract}

\section{INTRODUCTION}

Auxotrophic markers have proved among the most useful of those used in studying the genetics of micro-organisms. With knowledge of the minimum nutritional requirements of parent organisms one can hope to derive auxotrophic mutant strains and devise highly selective media capable of detecting rare recombinational events involving the genetic determinants of auxotrophy. As a preliminary to a study of the genetics of Pasteurella pseudotuberculosis we investigated the requirements of this species, as reported here. Since this species has many properties in common with $P$. pestis it was of interest to observe whether the resemblances between the two extended to the nutritional requirements. Accordingly we have re-examined some aspects of the nutrition of $\boldsymbol{P}$. pestis in parallel with $P$. pseudotuberculosis. In addition we also investigated strains of Pasteurella ' $\mathrm{X}$ ' (Knapp \& Thal, 1963), a species which, as yet, has not received intensive study but seems to have close relationship to $\boldsymbol{P}$. pseudotuberculosis. Recently Mollaret \& Lucas (1965) used the name $Y$ ersinia enterocolitica for Pasteurella ' $\mathrm{X}$ ' and have given references to other synonyms.

Unlike previous investigators who have examined the nutrition of pasteurellas (Rao, 1939, 1940; Berkman, 1942; Doudoroff, 1943; Herbert, 1949; Hills \& Spurr, 1952; Higuchi \& Carlin, 1958; Brownlow \& Wessman, 1960), we attempted to 
define nutritional factors which permit reliable growth of organisms isolated on agar surfaces rather than of inocula in liquid media. We chose to do so, first because solidified media have greater application to genetic studies and, secondly to ensure that we were observing the requirements of the majority population of strains rather than those of meiotrophic minorities, which in liquid cultures might overgrow and obscure the response of the majority.

\section{METHODS}

Organisms. Pasteurella pseudotuberculosis. Representatives of each of the 5 known serological types A, B, C, D, E (Thal, 1954) were examined in detail. They were the strains PBI/ + (type A), $16 \mathrm{II}(\mathrm{B}), 43 \mathrm{III}(\mathrm{C}), 32 \mathrm{IV}(\mathrm{D})$ and $25 \mathrm{~V}(\mathrm{E})$. We included also the strain $\mathrm{PB} 1 /$ - which is a VW - derivative of $\mathrm{PB} 1 /+$ (Burrows \& Bacon, 1960) and the strains MD 31 and Nielson which are of interest in being VW + strains of the relatively rare serotypes $C$ and $D$, respectively. The strain Nielson is an example of a VW + isolation from a human infection. Apart from MD 31 (see below), the above and other strains mentioned in the text were from our own culture collection.

Pasteurella pestis. The virulent strain MP6 and the avirulent (VW-) strain TS were used to identify requirements for growth at $37^{\circ}$. A culture of strain MP 6 recently passaged in mice was used to ensure minimum contamination of the culture with spontaneously arising avirulent derivatives. Seventy-three additional strains from our collection were examined for their ability to grow at $28^{\circ}$ on a defined medium which we had previously developed for use with strain Ts at this temperature (Burrows \& Bacon, 1954). Strains which differed nutritionally from this strain are referred to individually in the text.

Pasteurella ' $X$ '. Five strains were investigated. Two we received from $\mathrm{Dr} E$. Thal (Statens Veterinarmedicinska Anstalt, Stockholm), labelled 'Winblad Pp-X 346' and 'Tungelsta Pp-X 370', which we refer to as strains XA and XB, respectively. Three other strains $\times 66, \times 69$ and $\times 70$ were kindly provided (together with the $P$. pseudotuberculosis strain MD 31 above) by Dr R. R. Brubaker, (Fort Detrick, Frederick, Maryland, U.S.A.).

Media. BAB: blood agar base no. 2 (Oxo Limited) supplemented with $\mathrm{Na}_{2} \mathrm{~S}_{2} \mathrm{O}_{5}$ $0 \cdot 1 \mathrm{~g}$. $/$. was used as the complete medium. The different minimal media used for the identification of growth requirements were as follows.

MA: (g./l. distilled water) $\mathrm{K}_{2} \mathrm{HPO}_{4}, 10 \cdot 5 ; \mathrm{KH}_{2} \mathrm{PO}_{4}, 4 \cdot 5 ;\left(\mathrm{NH}_{4}\right)_{2} \mathrm{SO}_{4}, 1 \cdot 0$; sodium citrate, $0.5 ; \mathrm{MgSO}_{4} .7 \mathrm{H}_{2} \mathrm{O}, 0.1$; agar (Oxoid No. 3), 15; adjusted to $\mathrm{pH} 7.2$ and autoclaved at $115^{\circ}$ for $15 \mathrm{~min}$; glucose, $2 \cdot 0$, added after autoclaving.

MAS: this was medium MA to which was added $\mathrm{Na}_{2} \mathrm{~S}_{2} \mathrm{O}_{5}, 0 \cdot 1$, after autoclaving.

MASGF: this was medium MAS $+(\mathrm{mM})$ glycine, 1.0 ; DL-isoleucine, 0.5 ; DLvaline, 0.2 ; L-cystine, 0.2 ; DL-methionine, 0.2 ; DL-phenylalanine, 0.2 . The concentrations given are those of the $\mathrm{L}$-isomer of racemic mixtures.

Growth factors tested ( $\mu \mathrm{M}$ final concentration in media): the 20 amino acids, 200; the purines adenine, guanine, xanthine, hypoxanthine, 50 ; the pyrimidines cytosine, thymine, uracil, 50; haemin, 10; the vitamins inositol, pimelic acid, 100; choline chloride, 20; nicotinamide, $p$-aminobenzoic acid, 5; pyridoxine, 2 ; Ca pantothenate, riboflavine, thiamine, 1 ; folic acid, $0 \cdot 1$; biotin, 0.01 . 
The customary precautions about purity of chemicals and cleanliness of glassware were observed throughout. Disposable plastic Petri dishes containing $20 \mathrm{ml}$. medium agar were used in all experiments. Growth factors were added as separate sterile solutions to the melted agar media just before plates were poured.

Suspensions of organisms. All strains were stored as dried pellets (Stamp, 1947). For use pellets were streaked on $\mathrm{BAB}$ plates, incubated for 2 days at $28^{\circ}$ to confirm their purity and the resulting growth subcultured to BAB slopes. These were incubated for $17 \mathrm{hr}$ at $28^{\circ}$, the organisms suspended in phosphate buffer $(0.033 \mathrm{M}$, $\mathrm{pH} \mathrm{7 \cdot 0)}$ and diluted (c. $10^{6}$-fold) in buffer to give $2 \times 10^{3}$ organisms $/ \mathrm{ml}$. Of the final dilution, $\mathbf{0} \cdot 1 \mathrm{ml}$. samples were spread over the surfaces of complete agar media and of the defined minimal media under test.

Incubation. Effort was made to ensure correct incubator temperatures. For experiments at $28^{\circ}$ small variations in temperature did not influence the results, but for those conducted near $37^{\circ}$ the temperature was critical. For experiments at $37^{\circ}$ an incubator fitted with an air-circulating fan and housing a recording thermograph was used. Its thermostat was adjusted to give a minimum temperature of $37^{\circ}$; this setting gave a maximum of $38^{\circ}$. Plates incubated in $\mathrm{CO}_{2}$-enriched atmospheres were housed in sealable cylindrical containers, $25 \mathrm{~cm}$. diam, and $25 \mathrm{~cm}$. deep, and gassed with a mixture of $95 \%(\mathrm{v} / \mathrm{v})$ air $+5 \%(\mathrm{v} / \mathrm{w}) \mathrm{CO}_{2}$ for $5 \mathrm{~min}$. before sealing and placing in the incubator. The containers were re-gassed daily. All plates were examined daily and scored for macroscopically visible colonies over an 8-day period of incubation.

Criteria of growth. Defined media were considered satisfactory when they permitted reliable growth of the sparsely seeded organisms into macroscopically visible colonies ( $1.0 \mathrm{~mm}$. diam.) within 3 days of incubation (4 days for the slower-growing Pasteurella pestis) and yielded colony numbers similar to those given by equal samples of suspensions plated on the complete medium.

Abbreviations. Growth factor names are abbreviated in the tables according to current usage in publications on microbial genetics :

$\begin{array}{llll}\text { ala } \text { alanine } & \text { ile isoleucine } & \text { ser serine } \\ \text { asp aspartic acid } & \text { met methionine } & \text { thi thiamine } \\ \text { cys cystine } & \text { nic nicotinamide } & \text { thio thiosulphate } \\ \text { glu glutamic acid } & \text { pan pantothenate } & \text { thr threonine } \\ \text { gly } \text { glycine } & \text { phe phenylalanine } & \text { try tryptophan } \\ & & & \text { val valine }\end{array}$

\section{RESULTS}

Nutritional requirements for growth at $28^{\circ}$

Pasteurella pseudotuberculosis. In preliminary tests all strains grew on medium MASGF, a defined medium which we had used extensively for the growth of Pasteurella pestis at $28^{\circ}$. Simplification of this medium by the omission of all amino acids and of bisulphite did not impair its efficiency as a plating medium for strains of $\boldsymbol{P}$. pseudotuberculosis of types A, B or $\mathbf{E}$, which thus were independent of any growth factors. These strains plated on medium MA produced colonies $1 \mathrm{~mm}$. in diameter within 3 days and gave viable counts equal to, or frequently exceeding, those on complete medium. The strains 43 III, MD31 and 32 IV grew slowly and 
inconsistently on medium MA but supplementation of this medium with thiamine or pantothenate permitted their growth at a rate similar to that shown by the above non-exacting strains. The single additions of valine, glutamic acid or methionine to medium MA accelerated the growth of strains 43 III and 32 IV but less effectively than the single additions of thiamine or pantothenate. The ability of these strains to grow on medium MASGF, which does not contain these vitamins, indicated that they were replaceable by the amino acid supplements contained in this medium. The type D strain Nielson resembled the strains of types A, B and E in having no growth factor requirements, unlike the other type D strain, 32 IV. Cultures of strain $25 \mathrm{~V}$ contained a minority population which showed a thiamine- or pantothenatedependence.

Thus for $\boldsymbol{P}$. pseudotuberculosis medium MA supplemented with thiamine (or pantothenate) was a medium suitable for the growth of all tested strains and for the identification and characterization of auxotrophic derivatives of them. In other studies we have used this medium successfully for all these purposes.

Pasteurella pestis. The requirements of representatives of this species (strains TS and MP 6 and derivatives of them) had already been defined. Essential factors were cystine, phenylalanine and methionine, with glycine, valine and isoleucine as stimulatory factors (Burrows \& Bacon, 1954). To ensure full viable counts on agar surfaces from small inocula the addition of bisulphite, or haemin (Herbert, 1949) is necessary, even on complete media. In this study we examined the behaviour of several other strains to identify those requiring additional factors and those for which cystine, phenylalanine and/or methionine were dispensible. Strains having additional requirements were characterized by conventional auxanographic techniques. The majority of strains (49 of 73 tested) were nutritionally similar to strains Ts and MP 6; those which differed are shown in Table 1. Although strains independent of methionine or of phenylalanine were observed no strain appeared capable of dispensing with cystine and thus to be able to utilize $\mathrm{SO}_{4}{ }^{2-}$ as a sole source of sulphur. (We would note here that thiosulphate can effectively replace any stated requirement for cystine throughout this paper.)

\section{Table 1. Strains of Pasteurella pestis differing nutritionally} from $P$. pestis strain $T s$ at $28^{\circ}$

$P$. pestis strain $\mathrm{TS}$ requires cystine + methionine + phenylalanine

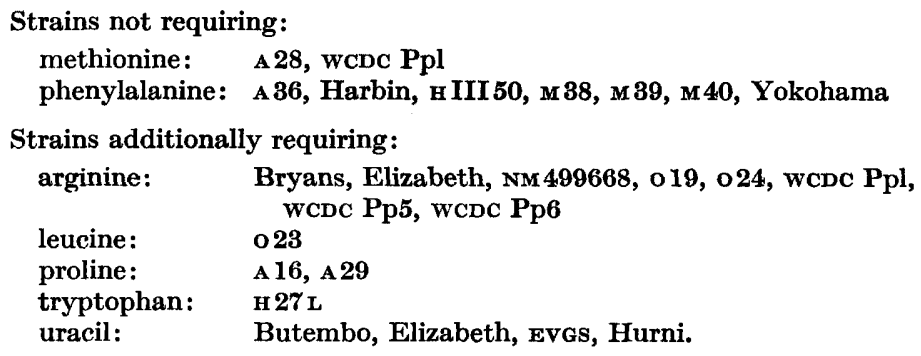

Pasteurella ' $X$ '. Strain $\mathrm{xB}$ and the majority components of cultures of $\mathrm{xA}$ and $x 66$ grew freely on medium MA and were therefore independent of growth factors. Tests with the minority component of strain XA (which we called strain XAb) showed 
it to be dependent on thiamine or valine. The finding that valine could replace thiamine was unexpected and led us to test the purity of our valine by examining its ability to support the growth of two thiamine-dependent mutant strains of $\boldsymbol{P}$. pseudotuberculosis and two of Salmonella typhi. None of these 4 mutants could use valine as an alternative to thiamine; all grew freely on medium MA + thiamine and on medium MA + thiamine + valine. We concluded that our valine was free from detectable thiamine and that it could in fact replace thiamine as a growth factor for strain XAb. In a further test each of 12 samples of valine from different sources showed growth factor activity for this strain. A sample of norvaline was however inhibitory and prevented growth even in the presence of valine or thiamine. Strain $\mathbf{x 7 0}$ resembled strain $\mathrm{xAb}$ in requiring thiamine or valine although here thiamine was the preferred factor. The minority component $\times 66 \mathrm{~b}$ of strain $\times 66$ required cystine + thiamine but seemed unable to use valine as a replacement for thiamine. We were informed by Dr R. R. Brubaker that strain $x 69$ was tryptophan-dependent and this we confirmed; it had no other requirements.

The growth factor requirements of the representatives of the 3 Pasteurella species for growth at $28^{\circ}$ are summarized in Table 2.

Table 2. Nutritional requirements for growth of Pasteurella spp. on agar media at $28^{\circ}$

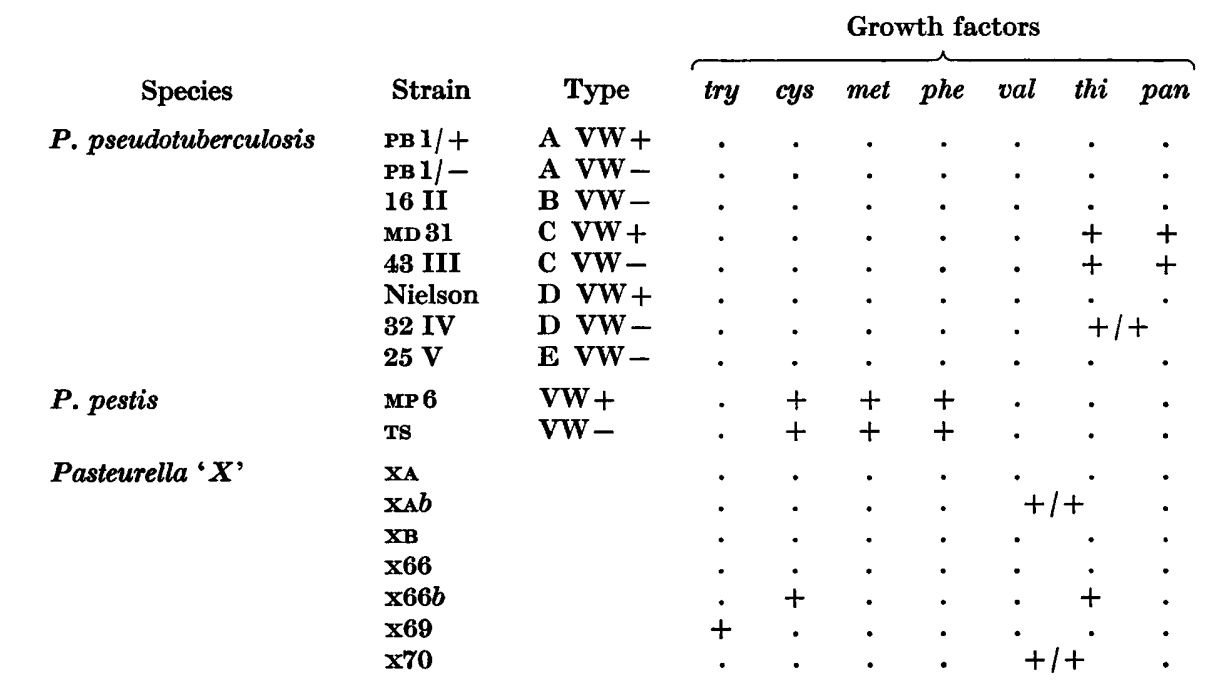

$+=$ growth factor required;.$=$ growth factor not required; $+1+=$ alternative factors.

\section{Nutritional requirements for growth at $37^{\circ}$}

Pasteurella pseudotuberculosis. The strains PB 1/ +, PB 1/-, 16 II, Nielson, MD 31 and $25 \mathrm{~V}$ behaved similarly and may be considered together. None could grow on medium MA or on medium MAS at $37^{\circ}$, but all grew to some extent, although not reproducibly or uniformly, on medium MASGF. Addition of pantothenate or of thiamine to this medium permitted reliable growth within 4 days. Omission of individual factors contained in medium MASGF + pantothenate again resulted in inconsistent growth, suggesting either that all components were essential or that 
some factors were inhibitory in the absence of others. At this point it seemed simpler to determine what additions to medium MA were necessary for growth. No single addition of the factors present in medium MASGF was effective. Addition of bisulphite (to give medium MAS) permitted some slow growth on the further addition of pantothenate or thiamine, but not on the addition of other factors singly. The further addition of cystine to medium MAS + pantothenate or thiamine improved the response of all strains whereas similar addition of methionine was inhibitory. Although no growth occurred on medium MAS + cystine the plated organisms retained their viability throughout 8 days of incubation and were capable of giving full colony numbers when subsequently re-incubated at $28^{\circ}$.

On a basal medium of MAS + cystine + pantothenate, thiamine and glutamic acid were effective supplements to promote early and reliable growth of all strains. Tests of medium MAS supplemented with all permutations of these 4 factors showed none to be effective when added singly,thiamine + glutamic acid to be the best of paired additions, and any triplet to be similarly effective to any other triplet and to all 4 factors together. These findings are shown in Table 3. Similar experiments with strains $\mathrm{PB} 1 /+$ and $\mathrm{PB} \mathbf{1} /$ - incubated in $\mathrm{CO}_{2}$-enriched air gave results comparable with those obtained above.

\title{
Table 3. Growth responses of some strains of Pasteurella pseudotuberculosis at $37^{\circ}$
}

\begin{abstract}
Basal medium was MAS. Organisms were plated at an estimated concentration of 200 organisms/plate; the plates were incubated at $37^{\circ}$ in air. Numbers of colonies are given only for those media on which colonies predominantly exceeded $1 \mathrm{~mm}$. diam. For other media: $\mathrm{s}=$ predominantly small colonies $($ c. $0.5 \mathrm{~mm}$. diam. $) ; \mathrm{m}=$ predominantly minute colonies $(c .0 .2 \mathrm{~mm}$. diam.); . = no visible colonies. The figures give the means of duplicate plates in each of three experiments.
\end{abstract}

Strain

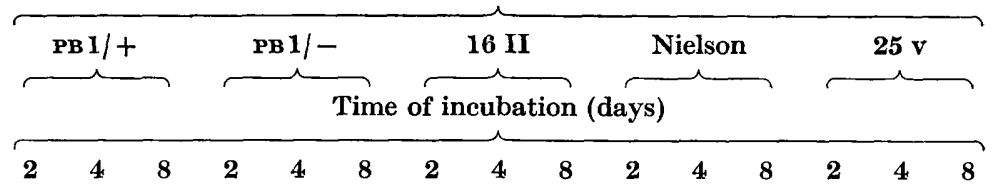

$\quad$ Medium
supplements
Nil
pan
cys
thi
glu
pan cys
pan thi
pan glu
cys thi
cys glu
thi glu
pan cys thi
pan cys glu
pan thi glu
cys thi glu
pan cys thi glu
(Complete
medium)

\begin{tabular}{|c|c|c|c|c|c|c|c|c|c|c|c|c|c|c|}
\hline \multicolumn{15}{|c|}{ no. of colonies } \\
\hline - & - & - & - & . & . & . & . & . & - & . & - & - & . & \\
\hline - & . & $\mathbf{m}$ & - & $\mathbf{m}$ & m & . & $\mathbf{s}$ & $\mathbf{s}$ & . & . & $\mathbf{s}$ & . & . & $\mathbf{m}$ \\
\hline . & . & . & . & . & . & . & . & . & . & . & . & . & . & . \\
\hline . & . & $\mathbf{s}$ & . & . & $\mathbf{s}$ & . & $\mathrm{m}$ & $\mathbf{s}$ & . & . & $\mathbf{s}$ & . & . & $\mathbf{s}$ \\
\hline - & . & m & . & . & $\mathbf{m}$ & . & $\mathbf{m}$ & m & - & . & m & . & . & $\mathbf{m}$ \\
\hline . & - & $\mathbf{s}$ & - & $\mathbf{m}$ & $\mathbf{s}$ & . & $\mathbf{s}$ & 95 & . & $\mathbf{m}$ & 126 & . & $\mathbf{m}$ & $\mathbf{m}$ \\
\hline . & . & $\mathbf{s}$ & . & $\mathbf{m}$ & $\mathbf{s}$ & . & $\mathbf{s}$ & 142 & . & . & 130 & - & $\mathbf{s}$ & 136 \\
\hline . & $\mathrm{m}$ & $\mathbf{s}$ & . & $\mathbf{s}$ & $\mathbf{s}$ & . & $\mathbf{s}$ & $\mathbf{s}$ & . & . & $\mathbf{s}$ & . & $\mathbf{s}$ & $\mathbf{s}$ \\
\hline . & 117 & 121 & . & 141 & 149 & . & . & 122 & . & . & 128 & . & . & 122 \\
\hline . & . & $\mathbf{s}$ & . & . & $\mathbf{s}$ & . & . & . & . & . & m & . & . & . \\
\hline & 154 & 154 & $\mathbf{s}$ & 173 & 193 & . & 165 & 165 & . & 173 & 177 & . & 173 & 173 \\
\hline . & 107 & 109 & $\mathbf{s}$ & 99 & 101 & $\mathbf{s}$ & 144 & 145 & . & 124 & 124 & 5 & 107 & 135 \\
\hline . & 122 & 131 & $\mathbf{s}$ & 146 & 151 & $\mathbf{s}$ & 140 & 161 & . & 76 & 116 & $\mathbf{s}$ & 115 & 122 \\
\hline . & 172 & 172 & $\mathbf{m}$ & 190 & 193 & . & 142 & 142 & . & 145 & 145 & . & 168 & 169 \\
\hline . & 168 & 168 & $\mathbf{m}$ & 192 & 192 & . & 129 & 154 & . & 161 & 161 & . & 154 & 159 \\
\hline . & 157 & 165 & $\mathbf{s}$ & 145 & 146 & $\mathbf{s}$ & 131 & 144 & . & 125 & 135 & $\mathbf{s}$ & 157 & 165 \\
\hline 59 & 162 & 162 & 177 & 177 & 177 & 146 & 146 & 145 & 165 & 165 & 165 & 153 & 153 & 153 \\
\hline
\end{tabular}


On a basal medium of MAS supplemented with thiamine, glutamic acid, cystine and pantothenate, we looked for a growth-stimulatory effect of calcium and of bicarbonate on the $\mathrm{VW}+$ strains $\mathrm{PB} / \mathrm{H}$ and Nielson. Comparable strains of Pasteurella pestis are reported to be selectively stimulated by these factors (Surgalla, Andrews \& Baugh, 1964). However, we did not detect such effect; additions of $\mathrm{CaCl}_{2}$ over the range $0.0025 \mathrm{M}$ to $0.02 \mathrm{M}$ showed progressively greater inhibition of growth with all strains, while $\mathrm{NaHCO}_{3}$ added over the same range was without detectable effect. The medium was not altered in its growth-producing efficiency by substituting glycerol as $\mathrm{C}$-source in place of glucose, or urea as $\mathrm{N}$-source in place of ammonium sulphate. Thiosulphate adequately replaced cystine as a source of sulphur for all strains, but slightly decreased the growth rate of strain $\mathrm{PB} 1 /+$. The mixture glycerol, ammonium sulphate and cystine tended to give more robust colonies after the complete 8 days of incubation than did other permutations of the alternative $\mathrm{C}$-, $\mathrm{N}$ - and S-sources.

The optimum $\mathrm{pH}$ value for growth on medium MAS + thiamine + glutamic acid + cystine + pantothenate medium was between $7 \cdot 0$ and $7 \cdot 2$. The minimum tolerated $\mathrm{pH}$ value was $6 \cdot 8$ and the maximum $9 \cdot 0$.

Pasteurella pseudotuberculosis strains 43 III and 32 IV behaved differently from those described above but similarly to each other. On the medium developed for the above strains few or no colonies developed within 8 days. Supplementation of this medium with aspartic acid, or with nicotinamide, permitted all plated organisms to produce visible colonies within 4 or 3 days, respectively. They therefore showed an alternative requirement for one or other of the factors aspartic acid or nicotinamide. Tests of our aspartic acid with two nicotinamide-dependent mutants of strain PB $\mathrm{I} /+$ showed that it was not detectably contaminated with nicotinamide. Thus a medium consisting of MAS + thiamine + glutamic acid + cystine + pantothenate + nicotinamide supported reliable growth of strains 43 III and $32 \mathrm{IV}$; omission of each factor singly showed each to be essential.

Pasteurella pestis. It was known that strains of this species had additional requirements for growth at $37^{\circ}$ and consequently would not grow on medium MASGF. Supplementation of this medium with biotin + pantothenate, with serine + threonine or with proline (factors which past workers have concluded to be essential for the growth of this organism at $37^{\circ}$ ), or with all these factors together, did not permit any colony formation. The further addition of glutamic acid to a medium containing all the above supplements sometimes allowed growth of a small fraction of the total plated organisms. Consistently reliable growth of both the strains TS and MP6 eventually was achieved on a medium comprising MASGF in which cystine was replaced by thiosulphate, with glutamic acid and thiamine added, followed by incubation in $\mathrm{CO}_{2}$-enriched air.

The requirement for additional $\mathrm{CO}_{2}$ was removed when the medium was supplemented with liver extract, with mouse blood or with casein hydrolysate. The activity of the last supplement suggested that an amino acid or acids could replace $\mathrm{CO}_{2}$ for growth and led to the identification of serine + threonine as an effective pair. It was necessary, however, to use serine at $0.02 \mathrm{~mm}$, i.e. one-tenth of the concentration routinely used, while keeping threonine at $0.2 \mathrm{~mm}$. Growth did not occur in air when these amino acids were both used at $0.2 \mathrm{~mm}$ but in the presence of additional $\mathrm{CO}_{2}$ the higher concentration of serine was not inhibitory. Later it was shown that 
the two amino acids could themselves be replaced by homoserine $0 \cdot 2 \mathrm{~mm}$. We would note here, however, that on some occasions poor growth was obtained with the above supplements whereas, in the same experiments, full growth resulted on unsupplemented plates incubated in $\mathrm{CO}_{2}$-enriched air; we therefore regard the latter condition as being the more reliable. Not infrequently pantothenate appeared to be able to replace thiamine, but we did not find conditions where such replacement was consistently effective and therefore regard thiamine as the more reliable growth factor.

We concluded from this part of our study that the most reliable defined medium for the growth of our representative strains of Pasteurella pestis was that consisting of medium MAS supplemented with the thiosulphate, glycine, valine, isoleucine, methionine, phenylalanine, glutamic acid, thiamine, $\mathrm{pH} 6 \cdot 8$ (see later), with incubation in $\mathrm{CO}_{2}$-enriched air. Whereas for growth in air glycine and glutamic acid appeared to be essential, their omission from media incubated with $\mathrm{CO}_{\mathbf{2}^{-}}$ enrichment resulted only in delayed initiation of growth. Omission of any other factor prevented growth.

On media containing serine + threonine reasonable growth occurred only over the range from $\mathrm{pH} 6 \cdot 4$ to $7 \cdot 0$ in air. For growth on the same medium in $\mathrm{CO}_{2}$-enriched air the $\mathrm{pH}$ value was less critical and high counts were obtained within 3 days over the tested range $\mathrm{pH} 6.0$ to $7 \cdot 2$ (Table 4 ). On media not containing serine and threonine the tolerated $\mathrm{pH}$ values for growth in $\mathrm{CO}_{2}$-enriched air were 6.4 minimum and $8 \cdot 2$ maximum; on all the tested media the optimum $\mathrm{pH}$ value appeared to be near to 6.8. It is probable that much of the inconsistent behaviour we experienced with

Table 4. Influence of $p H$ value on the growth of Pasteurella pestis strain ts on defined agar medium at $37^{\circ}$

Defined medium was MAS + thiosulphate + glycine + valine + isoleucine + methionine + phenylalanine + glutamic acid + serine (0.02 $\mathrm{mM})$ +threonine +thiamine. The medium was adjusted from its initial $\mathrm{pH} \mathbf{7 \cdot 2}$ to the required values by adding $\mathrm{N}-\mathrm{HCl}$. Plates were seeded with about 200 organisms, divided into two sets of which one was incubated in air and the other in air enriched with $\mathrm{CO}_{2}(5 \%, v / v)$ at $37^{\circ}$. The figures are the means of duplicate plates in each of two experiments.

\begin{tabular}{|c|c|c|c|c|c|c|}
\hline & & & & & & \\
\hline & & & & & $95 \%$ & $+5 \%$ \\
\hline & & & ae of $i$ & on $(d s$ & & \\
\hline $\begin{array}{c}\text { Defined } \\
\text { medium at }\end{array}$ & $\mathbf{3}$ & 4 & $\begin{array}{l}6 \\
\text { Col }\end{array}$ & $\stackrel{8}{\text { nbers }}$ & $\mathbf{3}$ & 8 \\
\hline $7 \cdot 2$ & 0 & 20 & 65 & 78 & 166 & 166 \\
\hline $7 \cdot 0$ & 54 & 141 & 161 & 165 & 189 & 189 \\
\hline $6 \cdot 8$ & 53 & 150 & 162 & 168 & 230 & 230 \\
\hline $6 \cdot 6$ & 37 & 91 & 149 & $153^{\circ}$ & 223 & 224 \\
\hline $6 \cdot 4$ & 33 & 128 & 160 & 161 & 236 & 236 \\
\hline $6 \cdot 2$ & 0 & 13 & 65 & 82 & 195 & 195 \\
\hline $6 \cdot 0$ & 0 & $\mathbf{3}$ & 42 & 52 & 182 & 193 \\
\hline $\begin{array}{l}\text { BAB comp } \\
\text { medium }\end{array}$ & 200 & 200 & 200 & 200 & 204 & 204 \\
\hline
\end{tabular}


Pasteurella pestis in experiments before the recognition of the beneficial effects of $\mathrm{CO}_{2}$ resulted from the use of defined media at $\mathrm{pH} 7 \cdot 2$ which is seen to be near the upper limit of tolerable $\mathrm{pH}$ value for growth in air.

It has been observed that virulent strains of Pasteurella pestis have an apparent dependence on $\mathrm{Ca}$ ions, not shown by $\mathrm{VW}$ - avirulent strains, since the former are inhibited when plated on complete media supplemented with $\mathbf{M g}$ ion +oxalate, whereas the latter grow freely, at $37^{\circ}$ (Higuchi \& Smith, 1961; Brubaker \& Surgalla, 1962). As we had not observed our representative strains to differ in growth factor requirements in this study it seemed of interest to examine their relative responses to Ca-deficiency on the defined medium now available. This contained $\mathrm{Mg}$ at $0.4 \mathrm{~mm}$ but no $\mathrm{Ca}$ other than that contaminating the ingredients. Of these the agar would have contributed the bulk of the $\mathrm{Ca}$ in the medium and, according to data supplied by the manufacturers, would have yielded a Ca concentration of 0.13 mM. We anticipated therefore that additions of $\mathrm{Na}$ oxalate in excess of this concentration would selectively inhibit the virulent representative strain MP 6 if this, in fact, had a requirement for Ca in excess of that of the VW - strain. No differential inhibition was however observed over a range of additions of $\mathrm{Na}$ oxalate from $\mathbf{0 . 2 5}$ $\mathrm{mm}$ to $20 \mathrm{~mm}$. Titration of the optimum addition of $\mathrm{Ca}$ to the medium showed this to be $4 \mathrm{~mm}$, and of $\mathrm{Mg} 8 \mathrm{~mm}$, for both strains. The single addition of Ca $20 \mathrm{~mm}$

Table 5. Comparative behaviour of Pasteurella pestis strains Ts (VW-) and MP6 $(V W+)$ on defined media variously supplemented with oxalate, $\mathrm{Mg}$ and $\mathrm{Ca}$

Complete medium was BAB. Defined medium was MAS + thiosulphate + glycine + valine + isoleucine + methionine + phenylalanine + glutamic acid + thiamine; pH 6.8. Supplements: $\mathrm{Ox}=\mathrm{Na}$ oxalate, $\mathrm{Mg}=\mathrm{MgCl}_{2}, \mathrm{Ca}=\mathrm{CaCl}_{2}$ each to give $20 \mathrm{mM}$ except where specified. To permit easier enumeration of colonies on the white opaque media resulting from the double supplementation with $\mathrm{Ox}$ and $\mathrm{Ca}$, triphenyl tetrazolium chloride was added to all plates to a final concentration of $0.005 \%$. Plates were incubated at $37^{\circ}$ in $95 \%(v / v)$ air $+5 \%(v / v) \mathbf{C O}_{2}$ and counted daily. Counts are expressed as $\%$ of the number of colonies developing on unsupplemented medium BAB. The figures give the means of duplicate plates in each of three experiments.

$\overbrace{\overbrace{3}^{4} \overbrace{4}^{T s} \text { Time of incubation (days) }}^{\text {Strain }} \overbrace{3}^{\text {MP 6 }}$

Medium Supplement(s)

Colony numbers (\%)

BAB

Defined

$$
\mathrm{Ox}+\dot{\mathrm{Mg}}
$$

Ox $2.5 \mathrm{~mm}$

Ox $5 \mathrm{~mm}$

Ox $10 \mathrm{~mm}$

$\mathrm{Mg}$

$\mathrm{Ca}$

$\mathrm{Ox}+\mathrm{Mg}$

$\mathrm{Ox}+\mathrm{Ca}$

$\mathrm{Mg}+\mathrm{Ca}$

$\mathrm{Ox}+\mathrm{Mg}+\mathrm{Ca}$

100
109
64
37
45
38
35
0
26
75
21
11

$\begin{array}{rrrrr}100 & 100 & 100 & 100 & 100 \\ 109 & 109 & 3 & 6 & 48 \\ 89 & 95 & 69 & 104 & 109 \\ 81 & 93 & \mathbf{7 4} & 106 & \mathbf{1 2 5} \\ 62 & \mathbf{8 6} & \mathbf{5 7} & \mathbf{8 3} & \mathbf{1 0 7} \\ \mathbf{5 7} & \mathbf{6 4} & \mathbf{5 9} & \mathbf{6 7} & \mathbf{1 0 5} \\ \mathbf{7 6} & \mathbf{9 4} & \mathbf{7 6} & \mathbf{8 0} & \mathbf{1 1 5} \\ 10 & \mathbf{7 4} & \mathbf{0} & \mathbf{2 1} & \mathbf{7 4} \\ \mathbf{9 6} & 114 & \mathbf{1 6} & \mathbf{5 1 *} & \mathbf{9 8} \\ \mathbf{8 7} & 105 & \mathbf{9 0} & \mathbf{1 2 0} & \mathbf{1 2 4} \\ \mathbf{2 9} & \mathbf{3 3} & \mathbf{1 9} & \mathbf{3 2} & \mathbf{3 9} \\ \mathbf{9 1} & 98 & \mathbf{0} & \mathbf{5 9} & \mathbf{9 5}\end{array}$

* All of 10 colonies taken at random proved virulent for mice. 
inhibited both strains similarly; $\mathrm{Mg}$ at this concentration showed no marked inhibition of either. The combination of oxalate $+\mathrm{Mg}$ both at $20 \mathrm{~mm}$ (as used in the differential medium of Higuchi \& Smith) showed some selective inhibition of the virulent strain but not to an extent which could be of diagnostic value (Table 5).

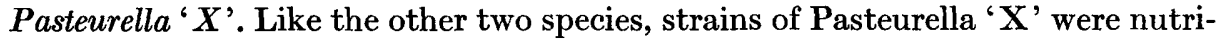
tionally more exacting at $37^{\circ}$ than at $28^{\circ}$. All showed a requirement for thiamine; at $37^{\circ}$ valine was not an effective alternative to this vitamin. These strains also showed impaired sulphur metabolism. Strain XA specifically required methionine, strains $\mathrm{x} 66$ and $\mathrm{x} 70$ cystine; the remaining strains $\mathrm{xB}$ and $\mathrm{x} 69$ required either methionine or cystine. We observed a requirement for alanine by strains $x 66$ and $\times 69$ which we had not observed with any other Pasteurella strain in this work. The substrain $\mathrm{x} 66 \mathrm{~b}$ in addition required glutamic acid + pantothenate + nicotinamide and thus resembled the strains $43 \mathrm{III}$ and $32 \mathrm{IV}$ of $\boldsymbol{P}$. pseudotuberculosis. Unlike these however substrain $\mathrm{x} 66 \mathrm{~b}$ could not use aspartic acid as an alternative to nicotinamide. Provision of additional $\mathrm{CO}_{2}$ did not alter the requirements of the strains of Pasteurella ' $\mathrm{X}$ '.

Table 6. Nutritional requirements for groroth of pasteurellas on ag ar media at $37^{\circ}$

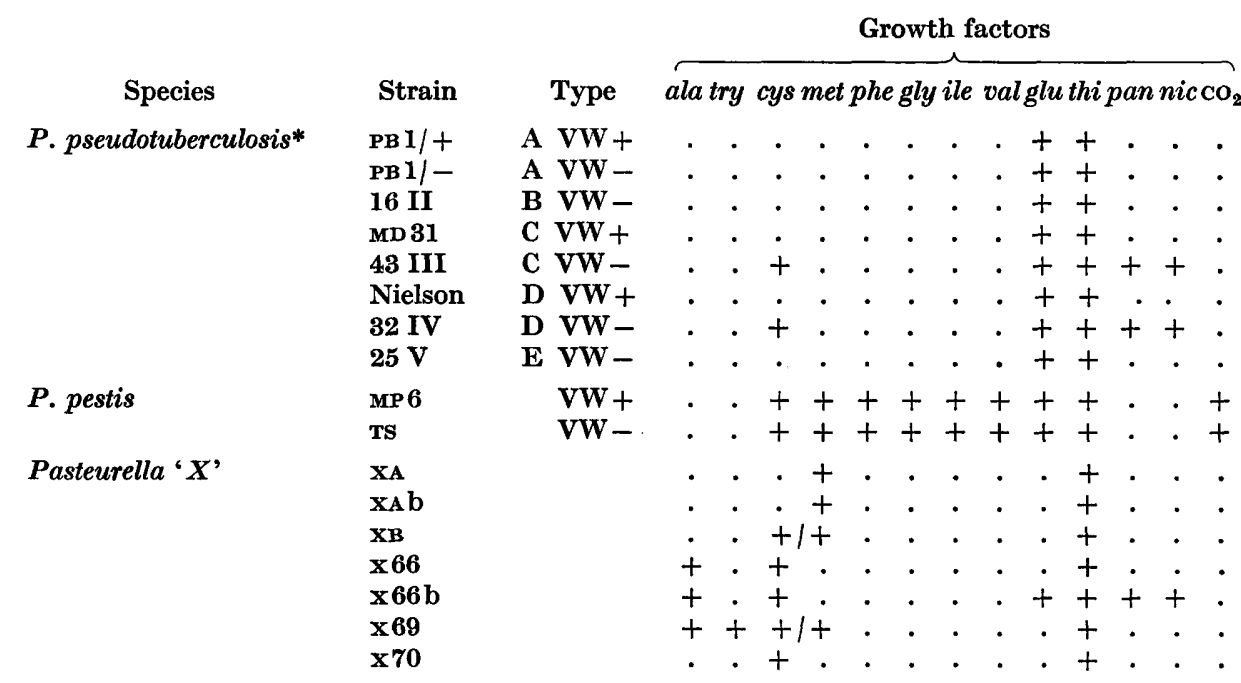

$+=$ growth factor required; . = growth factor not required; $+1+=$ alternative factors.

* Strains of $P$. pseudotuberculosis, other than $43 \mathrm{III}$ and $32 \mathrm{IV}$, grew well in the presence of any 3 of the 4 factors: $g l u, t h i, p a n$, cys. Of paired additions $g l u+t h i$ was preferred.

The optimum $\mathrm{pH}$ value determined for the growth of strain XA on MA + methionine + thiamine was about $\mathrm{pH} \mathbf{7 \cdot 8}$, but little difference in growth rate or in colony numbers was seen over the range $\mathrm{pH} 7 \cdot 2$ to 8.0. Although colonies developed less rapidly at higher $\mathrm{pH}$ values full colony numbers were obtained throughout the range $\mathrm{pH} \mathbf{7 \cdot 2}$ to $\mathbf{9 \cdot 0}$. In contrast, no colonies developed on media with initial $\mathrm{pH}$ values of 6.8 or lower.

The growth factor requirements of the representatives of the 3 Pasteurella species for growth at $37^{\circ}$ are summarized in Table 6. 


\section{DISCUSSION}

The strains of Pasteurella pseudotuberculosis examined differed from those of $P$. pestis for growth at $28^{\circ}$ in having no growth factor requirements, or a simple requirement for thiamine or pantothenate. This was so for 75 of 76 strains from our collection; the exception was $P$. pseudotuberculosis strain $74 \mathrm{R}$ which was dependent on additional, unidentified factor(s). It is however not difficult to select strains of $\boldsymbol{P}$. pestis which nutritionally resemble strains of $\boldsymbol{P}$. pseudotuberculosis in having no amino acid requirements by being phenylalanine- and methionine-independent and, like their parents, capable of utilizing thiosulphate in place of cystine. Such strains are still distinguishable from $P$. pseudotuberculosis by their inability to use $\mathrm{SO}_{4}{ }^{2-}$ as a sole source of sulphur (Englesberg, 1952). It is of interest to note that amino acid independent strains of $\boldsymbol{P}$. pestis isolated by us retain the high virulence characteristic of this species.

From our survey of the requirements of strains of Pasteurella pestis for growth at $28^{\circ}$ it is notable that those classified by Devignat (1951) as 'var. mediaevalis' (on criteria of geographical distribution, ability to ferment glycerol and inability to reduce nitrate) were all phenylalanine-independent. In this respect they differed from strains classified in the varieties 'antiqua' (glycerol + , nitrate + ) and 'orientalis' (glycerol - , nitrate +). Possibly the distinctive behaviour of the virulent strain Yokohama in the studies of Wessman, Miller \& Surgalla (1958) was associated with its independence of phenylalanine. A second notable point is that whereas Rao (1939) identified proline as an essential factor for the growth of $\boldsymbol{P}$. pestis, strains with this requirement seem to be rare; only two such strains were identified among the 73 examined here. Arginine-dependence seems to be more common among strains of recent isolation in North America (strains prefixed WCDC and NM) than among those isolated elsewhere.

Experiments conducted at $\mathbf{2 8}^{\circ}$ in the present work generally were reproducible and gave clear indications of the essential requirements for growth with all strains. This was by no means so for those conducted at $37^{\circ}$, particularly with Pasteurella pestis. One reason for the conflicting results with this organism became clear when the influence of $\mathrm{CO}_{2}$-enrichment was discovered. We were then able to show that growth or no growth on some defined media depended on the chance presence or absence of cultures being grown for other purposes in the incubator. Similarly, when plates of defined media were housed in containers (initially to prevent desiccation during prolonged incubation) growth could occur when complete medium control plates were housed in the same containers but not otherwise. By using $\mathrm{CO}_{2}$-enriched atmospheres and media at $\mathrm{pH} 6.8$ we removed most of the irregular behaviour earlier experienced with this organism. Strains of the other two species showed no requirement for additional $\mathrm{CO}_{2}$. Nevertheless, they were $\mathrm{CO}_{2}$-dependent as shown by their delayed growth and poor viability on incubation at $37^{\circ}$ in air from which $\mathrm{CO}_{2}$ had been removed by absorption with soda-lime, even when plated on complete media.

All three Pasteurella species were similar in having additional requirements for growth at $37^{\circ}$ over those required at $28^{\circ}$, as was first shown for Pasteurella pestis by Hills \& Spurr (1952). Impaired thiamine-synthesis was noted with all representatives. The interchangeability of aspartic acid and nicotinamide for the growth of 
some strains of $\boldsymbol{P}$. pseudotuberculosis accords with the findings of Ortega \& Brown (1960) and of Albertson \& Moat (1965) that the biosynthesis of nicotinic acid involves condensation of aspartic acid with a $3 \mathrm{C}$ compound.

On agar solidified media our virulent and avirulent strains of Pasteurella pestis showed equal dependence on added $\mathrm{CO}_{2}$ for growth; this contrasts with the results of others with liquid media where only virulent strains showed such dependence (Delwiche, Fukui, Andrews \& Surgalla, 1959; Surgalla, Andrews \& Baugh, 1964). In studies with cell-free extracts Baugh, Lanham \& Surgalla (1964) found that materials from virulent and from avirulent ( $V W-$ ) organisms equally were capable of fixing $\mathrm{CO}_{2}$ by carboxylation of phospho-enolpyruvate to give oxalacetate and thence aspartate. In tests which we have not detailed here neither of these products was capable of replacing $\mathrm{CO}_{2}$ for growth in air under our conditions. In similar contrast, we did not detect any marked difference in the calcium requirements of our two representatives whereas with liquid media, Higuchi, Kupferberg \& Smith (1959) noted a higher requirement on the part of virulent strains. Gadgil (1964), however, in studies with a virulent strain growing in liquid media observed a complex response to $\mathrm{Mg}$ and $\mathrm{Ca}$ additions which could not simply be explained in terms of the availability of $\mathrm{Ca}$ for growth. The selective inhibition of virulent strains on the addition of $\mathrm{Mg}$ oxalate to complete agar media (Higuchi \& Smith, 1961; Brubaker \& Surgalla, 1962), which is attributed to Ca-deficiency, may have another basis.

We gratefully acknowledge the interest and advice of Dr C. E. Gordon Smith and the excellent technical assistance of Mr W. Stott throughout this work.

\section{REFERENCES}

Albertson, J. N. \& Mont, A. G. (1965). Biosynthesis of nicotinic acid by Mycobacterium tuberculosis. J. Bact. 89, 540.

Baugh, C. L., Lanham, J. W. \& Surgalla, M. J. (1964). The effects of bicarbonate on growth of Pasteurella pestis. II. $\mathrm{CO}_{2}$ fixation into oxalacetate by cell-free extracts. J. Bact. 88, 553.

Berkman, S. (1942). Accessory growth factor requirements of the members of the genus Pasteurella. J. infect. Dis. 71, 201.

Brownlow, W. J. \& Wessman, G. E. (1960). Nutrition of Pasteurella pestis in chemically defined media at temperatures of $36^{\circ}$ to $38^{\circ} \mathrm{C}$. J. Bact. 79, 299.

Brubaker, R. R. \& Surgalla, M. J. (1962). Genotypic alterations associated with avirulence in streptomycin resistant Pasteurella pestis. J. Bact. 84, 615.

Burrows, T. W. \& Bacon, G. A. (1964). The basis of virulence in Pasteurella pestis: attempts to induce mutation from avirulence to virulence. Brit. J. exp. Path. 35, 129.

Burrows, T. W. \& Bacon, G. A. (1960). V and W antigens in strains of Pasteurella pseudotuberculosis. Brit. J. exp. Path. 41, 38.

Delwiche, E. A., Fukui, G. M., Andrews, A. W. \& Surgalla, M. J. (1959). Environmental conditions affecting the population dynamics and the retention of virulence of Pasteurella pestis: the role of carbon dioxide, J. Bact. 77, 355.

Devignat, R. (1951). Variétés de l'espèce Pasteurella pestis. Bull. Wld Hlth Org. 4, 247.

Doudoroff, M. (1943). Studies on the nutrition and metabolism of Pasteurella pestis. Proc. Soc. exp. Biol., N.Y. 53, 73.

Englesberg, E. (1952). The irreversibility of methionine synthesis from cysteine in Pasteurella pestis. J. Bact. 63, 675.

GaDGIL, M. D. (1964). Temperature sensitivity of $P$. pestis in relation to requirement of $\mathrm{Ca}^{++}$and $\mathrm{Mg}^{++}$. Indian J. Path. Bact. 7, 207. 
Herbert, D. (1949). Studies on the nutrition of Pasteurella pestis and factors affecting the growth of isolated cells on an agar surface. Brit. J. exp. Path. 30, 509.

Higuchi, K. \& Carlin, C. E. (1958). Studies on the nutrition and physiology of Pasteurella pestis. II. A defined medium for the growth of Pasteurella pestis. J. Bact. 75, 409.

Hrguchi, K. \& Smrth, J. L. (1961). Studies on the nutrition and physiology of Pasteurella pestis. VI. A differential plating medium for the estimation of the mutation rate to avirulence. J. Bact. 81, 605 .

Higuchi, K., Kupferberg, L. L. \& Smith, J. L. (1959). Studies on the nutrition and physiology of Pasteurella pestis. III. Effects of calcium ions on the growth of virulent and avirulent strains of $P$. pestis. J. Bact. 77, 317.

Hilus, G. M. \& SPURR, E. D. (1952). The effect of temperature on the nutritional requirements of Pasteurella pestis. J. gen. Microbiol. 6, 64 .

KnapP, W. \& ThaL, E. (1963). Untersuchungen über die kulturellbiochemischen, serologischen, tierexperimentellen und immunologischen Eigenschaften einer vorläufig 'Pasteurella $X$ ' benannten Bakterienart. Zentbl. Bakt. (I. Abt. Orig.), 190, 472.

Mollaret, H. H. \& Lucas, A. (1965). Sur les particularités biochemiques des sourches de Yersinia enterocolitica isolées chez les lièvres. Ann. Inst. Pasteur 108, 121.

Ortega, M. V. \& Brown, G. M. (1960). Precursors of nicotinic acid in Escherichia coli. $J$. biol. Chem. 235, 2939.

RAO, M. S. (1939). The nutritional requirements of the plague bacillus. Indian J. med. Res. 27,75 .

RAO, M. S. (1940). Further studies on the nutrition of the plague bacillus: the role of haematin and other compounds. Indian J. med. Res. 27, 833.

STAMP, Lond (1947). The preservation of bacteria by drying. J. gen. Microbiol. 1, 251.

Surgalla, M. J., Andrews, A. W. \& Baugh, C. L. (1964). Effects of bicarbonate on the growth of Pasteurella pestis. I. Differential response of virulent and avirulent cells. J. Bact. 88, 269.

Thal, E. (1954). Untersuchungen über Pasteurella pseudotuberculosis. Lund, Sweden: Berlingska Boktryckeriet.

Wessman, G. E., Miller, D. J. \& Surgalla, M. J. (1958). Toxic effect of glucose on virulent Pasteurella pestis in chemically defined media. $J$. Bact. 76, 368. 